\title{
Conrad Gesner, zoologiste
}

\section{Par Georges Petit}

Ce n'est point l'objet de cette conférence, que le Professeur Erwin AckerKNECHT m'a fait l'honneur de me demander, que de retracer la vie de Conrad Gesner. Cependant il m'apparaît nécessaire d'en rappeler les grandes lignes, du moins les principales étapes. Il est sans doute des existences qui s'identifient plus que la sienne à une œuvre. Mais la vie de celui dont on célèbre aujourd'hui le quatrième centenaire de la mort, cette vie, avec ses tribulations et comme ses incertitudes, fait apparaître l'œuvre plus considérable encore, plus immense, plus étonnante...

Ainsi Conrad Gesner est né à Zurich, le 26 mars 1516. Son père était marchand de fourrures. Nous avons écrit nous même que l'enfant fut peut-être attiré par la zoologie en voyant arriver dans la boutique paternelle les peaux d'animaux venues des Alpes et des contrées nordiques. Ce n'est pas impossible, car d'autres ont noté avec quel soin il devait décrire plus tard les espèces de ces régions.

La famille était pauvre; l'oncle maternel, Jean Frick, se chargea de l'éducation de Conrad Gesner. Il lui apprit les langues, la botanique et d'une manière générale lui fit prendre goût à tout ce qui touche la nature. C'est J. Jacques Ammann qui, à Zurich même, devait prendre le relai. Il guide l'intérêt de Conrad vers la médecine, où il devait encore s'initier avec Thomas Platter, qui était à la fois médecin et naturaliste.

Premier départ: Strasbourg. Ici Wolfgang Fabrice Capitan lui enseigne l'hébreu et lui prodigue des leçons sur la philologie. Ce fut ensuite Bourges. En effet, sa ville natale désirait que Gesner, et son ami Johannes Fries, ne demeurent point confinés à Zurich, mais apprennent à connaître «les mœurs et la sagesse des français».

Marguerite de Valois avait fondé dans cette ville une école qu'elle voulait célèbre et où elle attira CuJAs, que Toulouse et Cahors avaient, semble-t-il, sous-estimé. Précisément avec lui, Gesner devait s'initier à la jurisprudence, tout en regrettant que les sciences soient, à Bourges, quelque peu négligées ${ }^{1}$. Mais il était attiré par Paris où il put se rendre grâce à de nouveaux subsides venus de sa ville natale. Il y fit preuve d'une activité, sinon d'une avidité intellectuelle, considérable. Il se plonge dans la littérature gréco-latine et je ne sais plus qui devait le qualifier de «dévorateur de livres».

A Strasbourg, où il revint avant de regagner Zurich, il devait apparaître, déjà, comme «un prodige de savoir». Un secours du conseil d'Instruction de Zurich lui permet de se

1 Johannes Hanhart, de Winterthur, a publié en 1824 une contribution à l'histoire de la vie de Gesner. Dans la table générale des matières, pour le chapitre III, on lit «Ses études à Bourg en Bresse et à Paris». Cependant au cours du chapitre (p. 25), il est bien question du voyage à Bourges, «capitale du Berry». Dans la note 2 de la p. 27 nous trouvons la graphie: Bourges en Bresse. Cette confusion (Bourg en Bresse, Bourges et enfin Bourges en Bresse) est curieuse et méritait d'être signalée. 
rendre à Bâle pour continuer ses études médicales interrompues. Il cherche toujours à augmenter ses ressources et il entreprend alors une édition du Dictionnaire gréco-latin de Phavorinus Camers. Deux ans après, c'est Lausanne, où il enseigne le Grec à l'Académie. Cependant un nouveau subside lui permet de se rendre à Montpellier. Il y resta quatre mois. Ce séjour apparaît capital pour ce naturaliste jusqu'ici surtout linguiste, professeur de lettres, étudiant en médecine itinérant. Car il fréquenta dans cette ville Pierre Belon «consultissimus naturae Bellonius», a dit de lui Gesner - se lia d'amitié avec Rondelet ${ }^{2}$. Il fit aussi connaissance de ce curieux LAurent Joubert, professeur de médecine, qui écrit des vers à la gloire de Rondelet, avant de traduire son Histoire des Poissons, en langue «gallicane».

Nous verrons que son ouvrage sur les Poissons et les animaux aquatiques porte incontestablement la marque de la fréquentation de ces premiers «spécialistes», qui sont déjà des monographes.

Il est enfin reçu docteur en médecine à Bâle, en 1541. Il va exercer à Zurich, mais il enseigne en même temps la philosophie. Cependant il voyage encore. Il se rend à Vienne, à Venise où il examine les poissons de l'Adriatique, à Augsbourg, où l'attire sa soif d'érudition et où il note les titres de tous les ouvrages connus en hébreu, en grec et en latin.

Mais c'est bientôt la fin, une fin prématurée. Une épidémie de peste bubonique sévissait à Zurich en 1565. Il avait échappé à une première épidémie l'année précédente. Cette fois il se sent atteint et condamné. Il se fait transporter dans son cabinet, où il avait fait placer un lit, au milieu de ses collections, de ses livres, de ses manuscrits, ce cabinet, véritable musée, où il passait sans doute tant d'heures de labeur; c'est dans ce cadre qu'il voulait s'éteindre. Il mourut à l'âge de 49 ans, dans la nuit du 13 au 14 décembre 1565.

On dit qu'il avait fait appeler son élève et ami GASPAR WoLF à qui il léguait sa bibliothèque et ses manuscrits. Il lui confia sa collection botanique. ЈАсов FreUd a tout fait pour rassembler ce qu'avait laissé Gesner. La tâche fut rude si on juge, comme nous le verrons, par ce qui s'est passé pour les insectes...

Conrad Gesner est surtout célèbre par son œuvre zoologique, ce monument de documentation qu'est l'Histoire des Animaux. Mais ce qui étonne, c'est son extraordinaire érudition, non seulement l'érudition incluse dans de tels ouvrages, mais celle qui s'y ajoute et se manifeste dans d'autres.

En 1545, à vingt-huit ans, il entreprend cette Bibliothèque Universelle (Bibliotheca universalis), sorte d'encyclopédie du $\mathrm{XVI}^{\mathrm{e}}$ siècle, premier essai de répertoire bibliographique, les titres des ouvrages étant donnés en hébreu, en grec et en latin.

Ce n'est pas tout; auparavant il avait traduit un traité sur les syllogismes, publié la description du mont Pilate, près de Lucerne, un traité sur les eaux minérales d'Allemagne et de Suisse. Il avait traduit les Stances de StobÉe (1543), les Allégories de Don Crysosтомe sur Homère (1544), publié une édition, expurgée, de Martial (1544), d'Elien, celle-ci en 1556, ses Epistolae medicinales, une préface aux œuvres de GALIEN et à l'Histoire des Plantes de Tragus (JÉRôMe Bock)! Dans un mémoire paru en 1555 (Mithridates

2 Il y eût de nombreuses lettres échangées entre les deux amis, mais Gesner déplore que cette correspondance ait céssé, après que Rondelet se fut marié avec une femme beaucoup plus jeune que lui! 
seu de differentiis linguarum), il examine cent trente langues anciennes et modernes. Enfin, dans un ouvrage il admet que les fossiles sont des animaux pétrifiés, mais n'abandonne pas l'idée que ce sont peut-être aussi des formations exclusivement minérales ${ }^{3}$.

Conrad Gesner a donc beaucoup écrit dans des domaines fort divers. D'aucuns l'ont considéré comme un polygraphe, lié à son cabinet de travail. Rien n'est moins juste. Nous avons vu qu'il avait voyagé beaucoup pour parfaire ses connaissances, en acquérir toujours de nouvelles.

P.Delaunay a pu dire qu'on pouvait même le ranger parmi les précurseurs de l'alpinisme. Il herborisait dans les divers pays où il passait, collectionnait animaux et pierres, prenait des notes. Il avait pu constituer ainsi un véritable musée, à l'imitation de FéLix Platter avec lequel il faisait d'ailleurs des échanges. Il avait de nombreux correspondants, un peu dans tous les pays et jusqu'en Pologne. Il leur demandait des renseignements sur des animaux, accompagnés, si possible, de dessins. Comme il le dit lui-même, il aurait souhaité avoir vu tous les animaux dont il parle. Il se réjouit d'avoir observé à Nîmes un furet, et il faillit être mordu quelques jours avant sa mort par une vipère qu'il conservait vivante dans son cabinet.

Rappelons la lettre qu'il écrivait en 1557 à son ami GASPAR WoLF, alors à Montpellier. Il lui demande, à défaut de l'animal lui-même, des renseignements détaillés sur une espèce de lézard ou de salamandre qu'on nomme «Blandin» dans le midi de la France, animal se mouvant lentement, très venimeux, parsemé d'étoiles sur le dos. Il s'agit incontestablement d'un Gecko (Tarentola mauritanica). Crespon dans sa précieuse Faune méridionale (1844) désigne cet animal par les noms vulgaires de Blando ou Blendu. Cet animal, il le recevra du reste, puisqu'il écrit au sujet du Blandin, dans ses Quadrupèdes ovipares: "figura prior ad vinum expressa est».

Parmi ses correspondants citons: Melchior Guilland (Hollande), FABricius (Allemagne), John Kay (Caïus), qui lui envoie des coquillages, des dents de Scarus et auquel il demande le crâne entier, et convenablement désséché, d'un «chien de mer»; William Turner lui expédie des mâchoires de poissons. Le Révérend Père Thevet lui adresse des langues de serpents (glossopètres), recueillies à Malte, qui passaient pour un contrepoison très efficace et, cadeau plus intéressant, la peau d'un animal, l'Aüt, qu'il a bien décrit, ce qui permet de l'identifier au Paresseux (Bradypus tridactylus). Pierre Belon gratifie Gesner d'un squelette d'Engoulevent, cependant que de Pologne il reçoit des cornes d'Elan et de Bison.

3 De rerum fossilium, lapidum et gemmarum maxima figuris et similitudinibus, 1565. 
Une peau de Caïman lui servit pour la description de cet animal; il la tenait de Johannes Fenerus Pedemontanus, qui l'avait lui-même reçu d'Henrison Scotus, retour du Brésil.

Les voyages étaient longs, inconfortables, périlleux, Gesner n'a pas eu d'autre part une vie dont les soucis étaient exclus, bien au contraire. Alors que d'aptitudes intellectuelles, quelle volonté, sans doute quelle mémoire, que de dons brillants et variés, mais aussi quel écrasant labeur a dû être le sien!

C'est bien ce que traduit BoerhaAve en le qualifiant de monstrum eruditionis et Cuvier, en le désignant comme un prodige de travail, de savoir et de sagacité.

Du reste, il disait lui-même, que seul un travail acharné lui permettait de venir à bout de l'œuvre entreprise.

Gesner travailla essentiellement à son Histoire des Animaux, de 1545 à 1551, date à laquelle parut le premier volume consacré aux Quadrupèdes vivipares; en 1554 parut le deuxième volume concernant les Quadrupèdes ovipares. Le troisième, sur les Oiseaux, parut en 1555 et le quatrième sur les Poissons et les autres animaux aquatiques, en 1558. Cette encyclopédie zoologique ne comporte pas moins de 4500 pages in folio; elle est illustrée par plusieurs centaines de figures.

D'autres faits attestent encore l'ampleur de son extraordinaire labeur. En 1556, comme prélude à son grand travail sur les Poissons, il avait édité chez Andréas Gesner un ouvrage sur les animaux aquatiques où l'on trouvait les Halieuthiques d'Ovide, accompagnés de notes et de corrections et une liste en allemand des animaux d'eau cités jusqu'à Pline.

En 1548, bien avant la publication de ses Quadrupèdes vivipares, C. Gesner avait édité une manière de Pandecte où l'on trouvait, avec noms grecs, latins, allemands, anglais, français, la liste des animaux connus de lui (chapitre 11); les animaux déjà dessinés pour l'illustration de ses ouvrages étaient marqués d'un astérisque.

Gesner avait prévu un ouvrage sur les Insectes. Thomas Penny eût en main les matériaux accumulés par lui. Il possédait aussi les notes d'EdouARD Wотton. Au bout de quinze ans, il n'avait pas achevé la mise en œuvre de ces énormes documents. Après sa mort, un médecin de Londres, Thomas Moufet, succombait, au double sens du mot, sans avoir pu terminer un ouvrage qui ne parut qu'en 1634. Cependant Cuvier a pu dire que Moufet était pour les Insectes, ce que Gesner était pour les Quadrupèdes, et le nom de Gesner figure dans le titre comme un rappel de son rapport.

Un sixième livre sur les Serpents resta inachevé. 
La conception même de l'œuvre est curieuse et imposante. Les Epistolae nuncupatoriae figurent, en guise de préface, à son grand traité. Il y expose ses vues générales, le plan d'ensemble qu'il a conçu et ses projets.

Beaucoup de naturalistes sont venus de la médecine et certains ont apporté à la zoologie une contribution qui n'est pas négligeable. Gesner nous apprend, précisément, qu'il ne s'est voué à la médecine qu'en raison de ses rapports avec la philosophie naturelle.

Dès le frontispice de son premier livre, il rend grâce au Dieu, créateur et bon. Mais il fait l'éloge de la raison. Il lui assigne un rôle prépondérant; il s'agit d'un précepte universel et éternel, devant lequel s'incline l'expérience. Cette expérience ne fait que confirmer ce que la raison conçoit. Il n'y a pas d'expérience probante sans raisonnement.

On peut dire que Gesner fut le premier bibliographe. Ce n'est pas pour rien qu'il avait conçu sa Bibliotheca universalis; le souci de citer ses devanciers apparaît dès le début et tout au long de son œuvre zoologique. Plus de trois cents noms sont mentionnés dans le Catalogus authorum extantium. Notons, par exemple, qu'il cite Aristote, Elien, Varon, Columelle, Pline, Martial, Athénée, Albert le Grand, Vincent de Beauvais, mais aussi Michel Herr qu'il avait du connaître à Strasbourg et qui avait écrit un livre important et devenu rarissime sur les Quadrupèdes.

Chaque étude consacrée à un animal est structurée selon le même ordre: sept ou huit sections, qui sont désignées toujours par les premières lettres de l'alphabet. Dès l'abord, le linguiste apparaît; le nom de chaque espèce est donné en diverses langues, anciennes et modernes: latin grec, hébreu, français, allemand, anglais, espagnol, italien, persan, slave, et ses nomina hispanica apportent une intéressante nomenclature zoologique portuguaise. Voilà qui est extrêmement précieux, car on pouvait connaître ainsi à quel animal correspondaient les noms qui lui étaient donnés en plusieurs langues et on avait, de ce fait, une synonymie étendue et précise.

On passe ensuite à l'habitat, puis à la morphologie externe et à l'anatomie; le milieu est encore évoqué à propos d'une manière de physiologie où l'on trouve des données sur la durée de la vie, la croissance, l'époque de la fécondation, la parturition, le nombre de petits et où Gesner traîte aussi des maladies. Puis il s'agit des mœurs, des instincts, de la vie psychique (antipathie, sympathie, rapports avec l'homme). La zoologie appliquée constitue une autre section, c'est-à-dire le mode d'utilisation de l'animal, la chasse, l'élevage, les soins à donner. Viennent ensuite le rôle alimentaire de l'animal et aussi l'examen des remèdes qu'on en peut tirer. 
Enfin, dans une dernière section, qui fait figure de partie littéraire, c'est un extraordinaire fatras de renseignements: philologie, onomastique, noms d'animaux appliqués aux plantes, aux pierres, à des hommes, des femmes, des fleuves, des villes..., histoire littéraire, légendaire, proverbiale.

Gesner réalise son dessein de léguer une grande encyclopédie où l'on pourrait trouver tout ce que l'on sait à son époque, une grande encyclopédie dont les livres représenteraient à eux seuls une bibliothèque... et une bibliothèque destinée, comme l'indique le sous-titre de l'Histoire des Animaux, aux philosophes, médecins, philologues, poètes, à tous ceux qu'intéresse, la linguistique, pour qu'ils s'instruisent et se distraient tout à la fois. Il est tout de même singulier de constater que dans cette énumération ne figurent pas les naturalistes proprements dits!

Sans doute faut-il encore expliquer par ses tendances d'esprit et ses inclinations pour la bibliographe, le fait que Gesner range par lettre alphabétique les animaux cités dans ses ouvrages. Sans doute y voyait-il une façon pratique et rapide de se documenter, en faisant aussi de cette encyclopédie une manière de dictionnaire zoologique. Mais cela le conduit à placer l'Hippopotame entre l'Hippocampe et la Sangue (Hirudo), à faire précèder la Balane (Balanus) par la Baleine (Balaena), le Castor par la Carpe, alors qu'il est suivi des Cétacés.

Gesner ne pouvait manquer d'estimer qu'une telle manière de procéder était dépourvue de sens en ce qui concerne les affinités et il savait bien qu'il n'y avait là aucun aspect valable de classification. Peut-être s'est-il trouvé dérouté par la richesse et la complexité du monde animal et a-t-il choisi ce qui pouvait paraître commode aux lecteurs de ses ouvrages, épaissis par une énorme documentation. Mais qu'il connaisse le sens de la classification, qu'il en soit même préoccupé, n'en doutons pas, quand on sait qu'il proposa une classification des plantes basée sur la fleur et les fruits.

Si la notion de genre demeure incertaine, du moins utilise-t-il le mot et il a pu noter les caractères communs à plusieurs individus et les rapports qui les unissent. D'ailleurs, il corrige lui-même, et dès le premier volume de son Histoire des Animaux, cette conception si criticable sous l'angle scientifique. En effet, à un nom d'animal, Gesner accole, en abandonnant le principe de l'ordre alphabétique, des formes différentes dues au sexe, à l'âge, ou même des espèces voisines. Par exemple, à la suite des quinze pages in octavo, qu'il consacre à l'Ane (de Asino), il cîte et étudie de Hinno et Ginno, de Onagro ou Asino scythicis et Asino africanus. Il consacre 78 pages au Bœuf et à la Vache (Bos et Vacca) et l'on trouve immédiate- 
ment à la suite de Tauro, de Vitulo, et toute une série de Bovidés: de Bubalo, de Bisonte, de Bonasso, de Uro, l'Urus ou Aurochs (Bos primigenius).

On reconnaît encore la tendance bien fragile de considérer des ensembles naturels dans le volume sur les Oiseaux. Dans la monographie consacrée à Aquila, qui totalise trente pages, on trouve le Balbuzard et le Pygargue; sous le vocable Anas sont réunis les Canards et les Oiseaux plongeurs; sous celui d'Accipiter, les Faucons.

Il faut souligner que Gesner, pour la première fois, signale la présence, en Suisse, du Gypaete ou Vautour doré (Gypaëtus barbatus). Il a parfaitement décrit le Waldrof, qui est, selon lui, le Corvus sylvaticus. Il s'agit en réalité du Gerontias = Comatibis eremita, autrefois présent en Suisse, répandu dans tout le domaine méditerranéen, raréfié aujourd'hui et localisé dans les régions subdésertiques, depuis les îles du Cap-Vert jusqu'à la Mésopotamie. Gesner indique la répartition géographique de l'espèce telle qu'elle se présentait en 1555, mais aussi la coloration, les migrations et jusqu'au contenu stomacal!

Dans ce même volume on pourrait relever bien des erreurs, des confusions. Rappelons par exemple que le merle de roche (Monticola saxatilis) est confondu avec le merle bleu (Monticola solitarius) et qu'il était sans doute heureux d'avoir recours à l'ordre alphabétique, en présence, notamment, de la foule des Passereaux, qui l'a, semble-t-il, submergé.

Dans le volume sur les poissons et les animaux d'eau, qu'on pourrait appeler les «Aquatiles», Gesner a été, nous l'avons indiqué, largement inspiré par RoNDELET et par BELON. Il s'applique à reconnaître des différence d'un animal à l'autre, alors qu'ils appartiennent à une même forme et à les marquer par une appellation différente. Par exemple, il distingue l'Acipenser tel que le décrit Rondelet (Sturio primus), de l'Acipenser tel que le décrit Belon (Sturio secondus). Ainsi apparaît le début de la nomenclature binaire. Elle se précise. Par exemple le chapitre Gobione fluviatilis précède le chapitre de Gobione nigro (Gobio niger de Rondelet). Il désigne par un nom de genre et un nom d'espèce, toute une catégorie de Mésanges: Parus major, $P$. coeruleus, $P$. ater, etc.

Le chapitre sur le Dauphin (16 pages in folio) est très intéressant. On conçoit que tous les récits sur le comportement de cet animal, et notamment cette manière d'attirance qu'il manifeste vis-à-vis des hommes, y figurent ${ }^{4}$. Il se documentait sur la baleine auprès de son confrère Chapelain, très

4 Les plus récentes observations sur le comportement des Tursiops en captivité donnent au récit des anciens une incontestable base de véracité. 
savant docteur de Bayonne, qui avait eu l'occasion d'assister à des captures de baleines. Il s'agissait de la baleine des basques (Balaena biscayensis). Comme Rondelet, il distingue le Dauphin et le Marsouin. Il distingue encore la Baleine de Rondelet et la Baleine de Belon, dont parle aussi Aldrovende, qui selon les uns serait un Rorqual, selon les autres un Hyperoodon; il figure l'Orque et qualifie le Cachalot de Physalo bellua ou Physetere.

Un animal appelé Ziphius porte deux dents à la mâchoire inférieure et il y a lieu de se demander si la représentation, très peu zoologique dans l'ensemble qu'il nous en donne, ne figurait point uu Cétacé du genre Mesoplodon (Mesoplodon bidens).

Mais voici que dans les Icones Animalium, éditées en trois parties de 1553 à 1560 , le mot «ordre » apparaît et du même coup le groupement et la répartition des animaux sous ce vocable.

Il y a là, incontestablement un nouvel effort pour y voir clair, et sans doute aussi, pour aller plus loin qu'Aristote. Mais les conceptions de Gesner et les rapprochements auxquels il aboutit ne manquent point, très souvent, d'être singuliers, et une grande confusion demeure.

Quadrupèdes vivipares, de "nature calme», avec cornes ou sans cornes, s'opposent aux vivipares «féroces». Un singe, le Babouin, figure avec l'ours, le tigre, le lion et l'explication qu'on donne parfois de ce classement si aberrant, n'est point satisfaisante. Un troisième ordre comprend de petits carnassiers, qui voisinent avec les insectivores et accueillent un édenté, le Tatou.

Les Oiseaux (Icones avium, 1555), comprennent huit ordres.

Les Poissons occupent onze ordres sur les dix-sept que représentent les animaux aquatiques marins. Ils sont répartis selon leur habitat, surtout selon leur forme (comprimés, allongés, épineux), d'après leur squelette aussi, et la Baudroie reste voisine des raies et des torpilles, selon l'opinion de Rondelet. Les Cétacés constituent le douzième ordre, et on trouve avec eux les phoques et les tortues marines.

Nous ne pouvons ici nous plonger dans les cinq ordres d'Invertébrés marins distingués par Gesner. Nous isolerons cependant quelques données.

Les Mollusques sans coquilles (De mollibus dictis) comprennent les Céphalopodes. On a loué Gesner de leur rattacher le Nautile, malgré la présence d'une coquille, encore qu'il ait voulu sans doute parler de l'Argonaute.

Actinies, Balanes, Méduses et Aplysies y prennent place - l'Aplysie, que Dioscoride comparait déjà à un Calmar et que Rondelet classait effectivement parmi les Céphalopodes. 
Il est regrettable que Gesner ait fait l'économie d'un ordre qui aurait si bien convenu aux Echinodermes, alors qu'il les place dans les Mollusques Testacés!

Les Insectes marins comprennent de menus Crustacés et il est méritoire de voir figurer, parmi les Isopodes, un parasite de Poisson, de la famille des Cymothéidés qu'il nomme Asilus marinus. Mais avec ses insectes on trouve des Annélides, des Siponcles et même l'Hippocampe, qui fait figure de Vers!

On a loué Gesner d'avoir fait figurer dans ses Zoophytis marinis, les Pennatules, l'Alcyon ou main de mer, et aussi une colonie de Botrylles... (Uva marina).

Certes, nous retrouvons dans l'œuvre de Gesner, la galerie des animaux mythiques, fabuleux, qu'il concentre, en particulier, dans l'ordre de Cetis. On y voit notamment l'image du monstre en habit d'évèque avec mitre et ornements pontificaux. Il y en a bien d'autres, mais il faut souligner que Gesner, en les représentant, sacrifie pour ainsi dire aux habitudes et aux croyances de ses devanciers ou de ses contemporains, sans y croire vraiment lui-même.

Nous avons dit que Gesner avait illustré ses ouvrages par plusieurs centaines de figures. Beaucoup étaient dessinées de sa main; d'autres étaient dues à des artistes zurichois; Jean Asper, Jean Thomas, par exemple, et pour les Oiseaux Marcus Schroen. On retrouve de nombreux dessins empruntés à Rondelet pour les Poissons et à Belon pour les Oiseaux.

D'une manière générale la facture de ces dessins est bonne. Bien des animaux sont représentés pour la première fois; tels sont le Castor, le Lama, appelé par Gesner: Allocamelus, d'après le dessin d'un exemplaire de cet animal amené à Anvers en 1558. La figure de la Girafe a été empruntée au voyage de Georges von Breydenbach. Les Exotica de Clusius ont fourni celles du Sagouin, du Paresseux, du Tatou. Le Renard paraît ironique et malin. La figure du Corvus sylvaticus a grande allure. Si les figures du Tigre et de la Girafe sont médiocres, celle de la Taupe est bonne, comme celles du Crocodile, de la Tortue luth...

Certes, il y a des indications fantaisistes, parmi lesquelles le fait d'attribuer des élytres au Scorpion...

L'image du Rhinocéros est due à Albert Dürer. Elle est puissante comme l'animal lui-même. Mais, outre la corne nasale, l'artiste en a figuré une autre, plus petite, spiralée - et imaginaire - dans la région des vertèbres cervicales. 
On a pu croire qu'il s'agissait d'un rhinocéros d'Afrique à deux cornes... Mais DüRER a fait son dessin d'après un croquis et une description due à Valentin Fernandez d'un Rhinocéros d'Asie, arrivé à Lisbonne, offert qu'il était par le roi du Cambodge au roi du Portugal. Cette corne cervicale figurait-elle sur le croquis remis à l'artiste ? C'est probable. Pourtant l'article de Gesner ne parle lui-même que de la corne nasale pointue, que l'animal aiguise sur les pierres. Mais la seconde est présente sur la figure et c'est ce qui explique sans doute qu'il eût fallu attendre deux siècles pour que le rhinocéros d'Asie soit représenté sans corne cervicale.

Gesner eut d'ailleurs bien des déboires avec l'illustration de ses ouvrages. Dans une lettre à CAÏUs (1561; citée par A. Delaunay, 1963), il accuse son imprimeur de vendre à part les figures de ses Animalia, lesquelles, par surcroît, étaient peintes sans soin.

En vérité Gesner avait révé, sous une forme parfaitement définie et conçue par lui, d'une œuvre immense.

A 49 ans, il l'avait déjà accomplie, au prix d'un incroyable labeur. Et si l'Histoire Naturelle y occupe une place prépondérante, nous avons vu qu'elle n'était pas la seule.

CEvre immense, avons-nous dit, et touffue, à laquelle sont venues s'ajouter d'autres ouvrages, tels les Icones, et ils ne sont pas faits pour l'alléger et l'éclaircir, car ils viennent pour ainsi dire chevaucher l'œuvre essentielle.

Du vivant de Gesner, il y eût aussi plusieurs éditions qui apportaient des corrections, des données nouvelles, des dessins nouveaux, par exemple, la deuxième édition des Quadrupèdes vivipares, dédiée à la Reine EuızABETH et qui parut en 1560 .

Mais il y a beau temps que ses ouvrages sont délaissés. On admire davantage Gesner par l'exubérance de son œuvre que par ce qu'elle contient. On n'a plus le courage et le temps de la lire. Elle constitue pourtant un état inégalable des connaissances sur les animaux jusqu'au milieu du XVI ${ }^{\mathrm{e}}$ siècle, et l'érudition, qui s'y étale, offre, pour l'historien des Sciences, une documentation rare, pleine d'inattendus et bien souvent d'attraits. 